\title{
Distributed Model Predictive Control for Tracking: A Coalitional Clustering Approach
}

\author{
Paula Chanfreut $^{\circledR}$, José María Maestre ${ }^{\circledR}$, Senior Member, IEEE, Antonio Ferramosca ${ }^{\circledR}$, Member, IEEE, \\ Francisco Javier Muros ${ }^{\oplus}$, and Eduardo F. Camacho ${ }^{\circledR}$, Fellow, IEEE
}

\begin{abstract}
A coalitional robust model predictive controller for tracking target sets is presented. The overall system is controlled by a set of local control agents that dynamically merge into cooperative coalitions or clusters so as to attain an efficient trade-off between cooperation burden and global performance optimality. Within each cluster, the agents coordinate their inputs to maximize their collective performance, while considering the coupling effect with external subsystems as uncertainty. By using a tube-based approach, the overall system state is driven to the target sets while satisfying state and input constraints despite the changes in the controllers clustering. Likewise, feasibility and stability of the closed-loop system are guaranteed by tracking techniques. The applicability of the proposed approach is illustrated by an academic example.
\end{abstract}

Index Terms-Coalitional model predictive control, control by clustering, tracking, robust control.

\section{INTRODUCTION}

$\mathbf{I}$ $\mathrm{N}$ the last decades, distributed control schemes have gained attention due to the increasing size, complexity, and modularity requirements of many systems [1]. In essence, the overall control problem is divided into smaller pieces that are assigned to local controllers, also known as agents. Conversely to decentralized control, where no inter-controller communication is used, in distributed approaches the agents share information through a network to coordinate their control actions. In this context, the classical assumption is to consider a fixed communication network with static neighborhoods. By contrast, some recent works explore dynamic neighbourhoods by considering switching communication topologies [2], [3], time-varying partitioning [4], plug and play schemes [5], [6], and clustering [7]. In this work, we will focus on coalitional control [8], a clustering approach where the use of the communication resources is penalized to deter the control scheme from exchanging unnecessary information, leading to a time-varying degree of coordination. In this context, the local agents dynamically merge into disjoint clusters or coalitions whose members coordinate their control actions. The underlying goal of these schemes is to enable communication only among highly coupled agents so as to obtain a performance close to a fully coordinated system,

P. Chanfreut, J. M. Maestre, F. J. Muros and E. F. Camacho are with the Department of Systems and Automation Engineering, University of Seville, Spain (e-mails: \{pchanfreut, pepemaestre, franmuros, efcamacho\}eus.es)

A. Ferramosca is with the Department of Management, Information and Production Engineering, University of Bergamo, Italy (e-mail: antonio.ferramosca@unibg.it).

This work is supported by the Spanish Training Program for Academic Staff under Grant (FPU17/02653), the European Research Council Advanced Grant OCONTSOLAR (SI-1838/24/2018), and the Spanish MINECO Project C3PO (DPI2017-86918-R). but reducing the complexity of the control problem and the cooperation efforts, thus improving the scalability. This idea has interesting connections with cooperative game theory [9], [10] and has been extended to the well-known framework of model predictive control (MPC) [11], considering applications where the coupling varies in time and space, such as irrigation canals [12] and traffic freeways [13]. Similar approaches have also been recently proposed for dealing with large-scale power systems, e.g., in [14], a distribution network is split into control areas to solve a voltage regulation problem in an admissible time; in [2], the communication topology switches according to the oscillation modes; and in [15], a wind farm is split up into subsets of wind turbines according to the wake coupling effect.

Undoubtedly, MPC has become the accepted standard for complex control problems in the processes industry [16]. One of its most appealing features is the ability to drive the system state to a pre-fixed target fulfilling constraints. Nevertheless, if the desired setpoint changes, then the feasibility of the controller may be lost and the controller can fail to track the reference [17]. Several solutions are proposed in the literature to deal with this tracking problem [18], based on switching strategies [19], reference governors [20]-[22], or treating the target change as a disturbance to be rejected [17], [23]. In this article, we focus on the methodology presented in [24][26], which guarantees recursive feasibility and convergence to the admissible setpoints while providing an enlarged domain of attraction. In particular, the distributed strategies in [27], [28] are enhanced here in a coalitional framework. The main goal is to design a robust coalitional MPC for tracking, where both coupling and external disturbances are uncertain from a local standpoint. To this end, the robust MPC in [29] is employed. In this regard, the uncertain dynamics are addressed with a tube-based approach [30], [31], hence maintaining the real system in the neighborhood of the nominal predictions, and also allowing an independent control law computation for each cluster. See also [32], [33] for some fairly recent results on coalitional MPC tube-based schemes. The global system state is driven to a given target set, while satisfying the states and inputs constraints, and assuring recursive feasibility and stability despite the dynamic formation of coalitions. In the line of [8], a double sample rate strategy is used, where the clusters composition may be periodically changed.

The paper is organized as follows. In Section II, the problem setting is introduced. In Section III, the MPC for tracking sets approach is implemented in a coalitional fashion. The control scheme that assures feasibility and stability is presented in Section IV. Finally, simulation results and concluding remarks are respectively described in Sections V and VI. 


\section{PROBLEM FORMULATION}

In this section, we present the model used to describe the system dynamics and the communication infrastructure. Additionally, the control objective we address is introduced.

\section{A. System dynamics}

Consider a class of linear systems that can be partitioned into a set $\mathcal{N}=\{1,2, \ldots, N\}$ of coupled subsystems, whose dynamics are modeled as ${ }^{1}$

$$
\begin{aligned}
& x_{i}^{+}=A_{i i} x_{i}+B_{i i} u_{i}+w_{i}, \\
& w_{i}=\sum_{j \in \mathcal{N}_{i}}\left[A_{i j} x_{j}+B_{i j} u_{j}\right]+d_{i},
\end{aligned}
$$

where $x_{i} \in \mathbb{R}^{n_{x_{i}}}$ and $u_{i} \in \mathbb{R}^{n_{u_{i}}}$ are respectively the state and input vectors of subsystem $i \in \mathcal{N}, x_{i}^{+} \in \mathbb{R}^{n_{x_{i}}}$ is its successor state, and $w_{i} \in \mathbb{R}^{n_{x_{i}}}$ aggregates the coupling among the state of agent $i$ and its set of neighbors, defined as $\mathcal{N}_{i}=\left\{j \in \mathcal{N} \backslash\{i\} \mid\left[A_{i j}, B_{i j}\right] \neq \mathbf{0}\right\}$, and external disturbances $d_{i} \in \mathbb{R}^{n_{x_{i}}}$. Matrices $A_{i j}$ and $B_{i j}$ are, respectively, the state transition and the input-to-state matrices for all $i, j \in \mathcal{N}$, and $\mathbf{0}$ represents a null matrix of the corresponding size. Accordingly, the global behavior can be modeled by matrices $A_{\mathcal{N}}=\left(A_{i j}\right)_{i, j \in \mathcal{N}}$ and $B_{\mathcal{N}}=\left(B_{i j}\right)_{i, j \in \mathcal{N}}$, which aggregate (1) for the $N$ subsystems, i.e.,

$$
x_{\mathcal{N}}^{+}=A_{\mathcal{N}} x_{\mathcal{N}}+B_{\mathcal{N}} u_{\mathcal{N}}+w_{\mathcal{N}}
$$

where $x_{\mathcal{N}}=\left[x_{i}\right]_{i \in \mathcal{N}}$ and $u_{\mathcal{N}}=\left[u_{i}\right]_{i \in \mathcal{N}}$ are the global state and input vectors, respectively. Additionally, $w_{\mathcal{N}}=\left[d_{i}\right]_{i \in \mathcal{N}}$ is the overall disturbance vector, which contains only external disturbances because all subsystems interactions are accounted for in matrices $A_{\mathcal{N}}$ and $B_{\mathcal{N}}$.

\section{B. Network structure}

The $N$ subsystems are individually governed by a set of local agents interconnected through a configurable communication network, i.e., the connections between agents can be dynamically either enabled or disabled. Let $\mathcal{G}=(\mathcal{N}, \mathcal{L})$ be the graph describing the network, where the nodes in $\mathcal{N}$ represent the local agents, and set $\mathcal{L}$ contains the links, i.e., $\mathcal{L} \subseteq \mathcal{L}^{\mathcal{N}}=\{\{i, j\} \mid\{i, j\} \subseteq \mathcal{N}, i \neq j\}$. Also, let $|\mathcal{L}|$ be the cardinality of $\mathcal{L}$, then we can derive a set $\mathcal{T}$ composed of $2^{|\mathcal{L}|}$ communication topologies $\Lambda \subseteq \mathcal{L}$, which are defined by the sets of enabled links, i.e.,

$$
\mathcal{T}=\{\overbrace{\Lambda_{0}}^{\Lambda_{\text {dec }}}, \Lambda_{1}, \ldots, \overbrace{\Lambda_{2}|\mathcal{L}|-1}^{\Lambda_{\text {cen }}}\},
$$

where $\Lambda_{\text {dec }}$ and $\Lambda_{\text {cen }}$ respectively refer to the decentralized and centralized configurations, i.e., $\Lambda_{\text {dec }}=\emptyset$ and $\Lambda_{\text {cen }}=\mathcal{L}$. In this respect, the term decentralized denotes the case in which the agents do not share any data, hence their knowledge is limited to local information, while centralized refers to communication using all available resources.

\footnotetext{
${ }^{1}$ For simplicity, the dependence with time step has been omitted along the full manuscript. Throughout this paper, superscript ' + ' is used for variables associated to time instant $k+1$ and no superscript refers to time $k$.
}

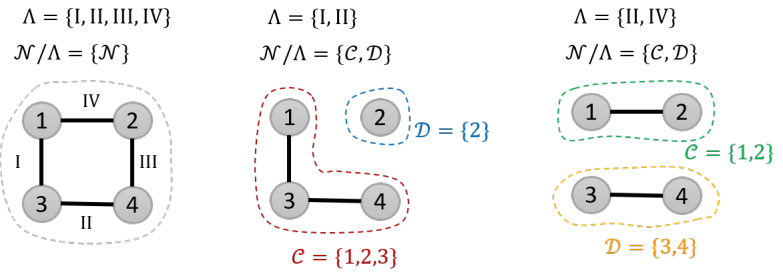

Fig. 1: Examples of topologies and coalitions for a network with 4 agents and 4 links. Letters $\mathcal{C}$ and $\mathcal{D}$ denote coalitions of agents, i.e., $\mathcal{C}, \mathcal{D} \in \mathcal{N} / \Lambda$ with $\mathcal{C} \cap \mathcal{D}=\emptyset$. Note that in the left figure all agents can communicate (see Remark 1).

Each $\Lambda \in \mathcal{T}$ arranges the $N$ subsystems into clusters or coalitions $\mathcal{C}$, i.e., agents connected by a path of enabled links. In this regard, the partition induced by $\Lambda$ over $\mathcal{N}$ is denoted as $\mathcal{N} / \Lambda$. Note that the number of clusters in $\mathcal{N} / \Lambda$ ranges from one, when all agents are connected $\left(\mathcal{N} / \Lambda_{\text {cen }}=\mathcal{N}\right)$, to $N$, corresponding to a fully decentralized system $\left(\mathcal{N} / \Lambda_{\text {dec }}=\right.$ $\{\{1\},\{2\}, \ldots,\{N\}\})$. An easy example with three different topologies following this notation is depicted in Fig. 1.

Remark 1: Hereafter, we consider that the communication links are bidirectional and assume indirect connectivity, i.e., any agent $i$ can send/receive data to/from agent $j$ if there exists a path of enabled links between $i$ and $j$.

1) Coalitional model: Dynamically, any coalition $\mathcal{C} \in$ $\mathcal{N} / \Lambda$ can be considered as a single system modeled by

$$
\begin{aligned}
& x_{\mathcal{C}}^{+}=A_{\mathcal{C C}} x_{\mathcal{C}}+B_{\mathcal{C C}} u_{\mathcal{C}}+w_{\mathcal{C}}, \\
& w_{\mathcal{C}}=\sum_{\mathcal{D} \in \mathcal{N}_{\mathcal{C}}}\left[A_{\mathcal{C D}} x_{\mathcal{D}}+B_{\mathcal{C D}} u_{\mathcal{D}}\right]+d_{\mathcal{C}},
\end{aligned}
$$

where $x_{\mathcal{C}}=\left[x_{i}\right]_{i \in \mathcal{C}} \in \mathbb{R}^{n_{x_{\mathcal{C}}}}$ and $u_{\mathcal{C}}=\left[u_{i}\right]_{i \in \mathcal{C}} \in \mathbb{R}^{n_{u_{\mathcal{C}}}}$ are respectively the aggregates of the states and inputs of subsystems $i \in \mathcal{C}$, and matrices $A_{\mathcal{C C}}$ and $B_{\mathcal{C C}}$ map the current coalition members states and inputs to their successor state. Similarly, $w_{\mathcal{C}}$ models the effect of neighboring coalitions $\mathcal{D} \in$ $\mathcal{N}_{\mathcal{C}}$ and the external disturbances on subsystems $i \in \mathcal{C}$. Matrices $A_{\mathcal{C D}}, B_{\mathcal{C D}}$ and set $\mathcal{N}_{\mathcal{C}}$ are defined analogously to the case of interacting subsystems.

\section{Objective statement and assumptions}

From now on, we consider the assumptions described below:

(i) The agents in $\mathcal{N}$ should drive the global system state to a target set $\Gamma_{\mathcal{N}}$ while satisfying state and input constraints.

(ii) The agents can dynamically merge into disjoint clusters $\mathcal{C}$ to compute their control inputs.

(iii) Each cluster $\mathcal{C}$ can measure $x_{\mathcal{C}}$ and has full knowledge of matrices $A_{\mathcal{C C}}$ and $B_{\mathcal{C C}}$, whereas the inter-coalitions coupling and external disturbances are uncertain.

Also, the following considerations are explicitly assumed:

Assumption 1: For any coalition $\mathcal{C}$ :

- Pair $\left(A_{\mathcal{C C}}, B_{\mathcal{C C}}\right)$ is stabilizable and state $x_{\mathcal{C}}$ is measurable.

- The state and input are constrained by

$$
x_{\mathcal{C}} \in \mathcal{X}_{\mathcal{C}}=\bigotimes_{i \in \mathcal{C}} \mathcal{X}_{i}, \quad u_{\mathcal{C}} \in \mathcal{U}_{\mathcal{C}}=\bigotimes_{i \in \mathcal{C}} \mathcal{U}_{i}
$$


where $\mathcal{X}_{\mathcal{C}}$ and $\mathcal{U}_{\mathcal{C}}$ are polytopic sets containing the origin in their interior, and $\mathcal{X}_{i}$ and $\mathcal{U}_{i}$ represent the constraint sets of the subsystems inside the coalition.

- Disturbances $w_{\mathcal{C}}$ are bounded by a convex compact polytope $\mathcal{W}_{\mathcal{C}}$ containing the origin in its interior, i.e.,

$$
w_{\mathcal{C}} \in \mathcal{W}_{\mathcal{C}}=\bigoplus_{\mathcal{D} \in \mathcal{N}_{\mathcal{C}}}\left[A_{\mathcal{C D}} \mathcal{X}_{\mathcal{D}}+B_{\mathcal{C D}} \mathcal{U}_{\mathcal{D}}\right] \oplus \mathcal{E}_{\mathcal{C}}
$$

where $\mathcal{E}_{\mathcal{C}}=\bigotimes_{i \in \mathcal{C}} \mathcal{E}_{i}$, being $\mathcal{E}_{i}$ a set containing all possible realizations of the external disturbances on subsystem $i$.

- There exist a feedback gain $K_{\mathcal{C}}^{\mathrm{e}}$ such that matrix $\left(A_{\mathcal{C C}}+\right.$ $\left.B_{\mathcal{C C}} K_{\mathcal{C}}^{\mathrm{e}}\right)$ is Schur [34], and a robust positively invariant set $\mathcal{R}_{\mathcal{C}}$ that satisfies

$$
\begin{aligned}
& \left(A_{\mathcal{C C}}+B_{\mathcal{C C}} K_{\mathcal{C}}^{\mathrm{e}}\right) \mathcal{R}_{\mathcal{C}} \oplus \mathcal{W}_{\mathcal{C}} \subseteq \mathcal{R}_{\mathcal{C}}, \\
& \mathcal{R}_{\mathcal{C}} \subset \operatorname{int}\left(\mathcal{X}_{\mathcal{C}}\right) \\
& K_{\mathcal{C}}^{\mathrm{e}} \mathcal{R}_{\mathcal{C}} \subset \operatorname{int}\left(\mathcal{U}_{\mathcal{C}}\right)
\end{aligned}
$$

Assumption 2: The global target set is defined as $\Gamma_{\mathcal{N}}=$ $\bigotimes_{i \in \mathcal{N}} \Gamma_{i}$, where $\Gamma_{i}$ are convex polytopic sets defining the target region for the state of subsystem $i$, that is,

$$
\Gamma_{i}=\left\{x_{i} \in \mathbb{R}^{n_{x_{i}}} \mid G_{\Gamma_{i}} x_{i} \leq g_{\Gamma_{i}}\right\},
$$

with $G_{\Gamma_{i}}$ and $g_{\Gamma_{i}}$ providing the H-representation of $\Gamma_{i}$. Accordingly, $\Gamma_{\mathcal{C}}=\bigotimes_{i \in \mathcal{C}} \Gamma_{i}$ is the target set of coalition $\mathcal{C}$. $\square$

Assumption 3: For any coalition $\mathcal{C}$, sets $\mathcal{R}_{\mathcal{C}}$ and $\Gamma_{\mathcal{C}}$ are such that $\Gamma_{\mathcal{C}} \ominus \mathcal{R}_{\mathcal{C}} \neq \emptyset$.

\section{Tracking Sets in a CoAlitional Framework}

The robust tube-based MPC for tracking [29] is characterized by:

(i) The introduction of an artificial steady state and input as optimization variables in an MPC problem based on the nominal dynamics, which allows for an extension of the domain of attraction of the MPC controller [24].

(ii) The use of an offset cost function that penalizes the deviation between the artificial and the real setpoints.

(iii) The use of a control law comprising an optimal input governing the nominal dynamics and an ancillary feedback controller to keep the real system in the neighborhood of the nominal predictions [30], [31], [35].

\section{A. Nominal steady state and input}

The nominal model of each cluster is derived from (4) by neglecting the effect of $w_{\mathcal{C}}$, that is,

$$
\bar{x}_{\mathcal{C}}^{+}=A_{\mathcal{C}} \bar{x}_{\mathcal{C}}+B_{\mathcal{C C}} \bar{u}_{\mathcal{C}},
$$

where $\bar{x}_{\mathcal{C}}$ and $\bar{u}_{\mathcal{C}}$ represent respectively the nominal state and input of $\mathcal{C}$. Accordingly, the set of possible nominal steady states and inputs for a certain cluster $\mathcal{C}$ can be derived from

$$
\left[\begin{array}{ll}
A_{\mathcal{C C}}-\mathbf{I}_{n_{x_{\mathcal{C}}}} & B_{\mathcal{C C}}
\end{array}\right]\left[\begin{array}{l}
\bar{x}_{\mathcal{C}}^{\mathrm{s}} \\
\bar{u}_{\mathcal{C}}^{\mathrm{s}}
\end{array}\right]=\mathbf{0}_{n_{x_{\mathcal{C}} \times 1}},
$$

where $\mathbf{I}_{n_{x_{\mathcal{C}}}}$ and $\mathbf{0}_{n_{x_{\mathcal{C}}} \times 1}$ represent respectively the identity matrix of dimensions $n_{x_{\mathcal{C}}} \times n_{x_{\mathcal{C}}}$ and the null matrix of dimensions $n_{x_{\mathcal{C}}} \times 1$. Therefore, the null space of matrix $\left[A_{\mathcal{C C}}-\mathbf{I}_{n_{x_{\mathcal{C}}}} B_{\mathcal{C C}}\right]$ allows a parametrization of the set of steady states in terms of a single variable in $\mathbb{R}^{n_{u_{\mathcal{C}}} \times 1}$ (see [24]).

\section{B. Invariant set for tracking}

Consider that the nominal coalitional dynamics (9) are controlled by a linear feedback control law, defined as

$$
\bar{u}_{\mathcal{C}}=\bar{u}_{\mathcal{C}}^{\mathrm{s}}+\bar{K}_{\mathcal{C}}\left(\bar{x}_{\mathcal{C}}-\bar{x}_{\mathcal{C}}^{\mathrm{s}}\right),
$$

where $\bar{K}_{\mathcal{C}}$ is a constant matrix that yields closed-loop stability of system (9) and satisfies

$$
\bar{P}_{\mathcal{C}}-\left(A_{\mathcal{C C}}+B_{\mathcal{C C}} \bar{K}_{\mathcal{C}}\right)^{\mathrm{T}} \bar{P}_{\mathcal{C}}\left(A_{\mathcal{C C}}+B_{\mathcal{C} C} \bar{K}_{\mathcal{C}}\right)=Q_{\mathcal{C}}+\bar{K}_{\mathcal{C}}^{\mathrm{T}} R_{\mathcal{C}} \bar{K}_{\mathcal{C}},
$$

being $\bar{P}_{\mathcal{C}}$ a positive definite matrix. Also, let us introduce the augmented system

$$
\left[\begin{array}{c}
\bar{x}_{\mathcal{C}} \\
\bar{x}_{\mathcal{C}}^{\mathrm{s}} \\
\bar{u}_{\mathcal{C}}^{s}
\end{array}\right]^{+}=\left[\begin{array}{ccc}
A_{\mathcal{C C}}+B_{\mathcal{C C}} \bar{K}_{\mathcal{C}} & -B_{\mathcal{C C}} \bar{K}_{\mathcal{C}} & B_{\mathcal{C C}} \\
\mathbf{0}_{n_{x_{\mathcal{C}}}} & \mathbf{I}_{n_{x_{\mathcal{C}}}} & \mathbf{0}_{n_{u_{\mathcal{C}}}} \\
\mathbf{0}_{n_{x_{\mathcal{C}}}} & \mathbf{0}_{n_{x_{\mathcal{C}}}} & \mathbf{I}_{n_{u_{\mathcal{C}}}}
\end{array}\right]\left[\begin{array}{c}
\bar{x}_{\mathcal{C}} \\
\bar{x}_{\mathcal{C}}^{\mathrm{s}} \\
\bar{u}_{\mathcal{C}}^{\mathrm{s}}
\end{array}\right],
$$

whose state is formed by the nominal coalitional state and its steady state and input. Considering this, in the tracking optimization problem the terminal set is defined as an invariant set $\bar{\Omega}_{\mathcal{C}}^{\mathrm{a}}$ of augmented system (13), i.e., the terminal set is an invariant set for any possible nominal equilibrium point, and contains all nominal initial states (and steady states) that can be stabilized by (11) while satisfying constraints (5) (see [24]).

Remark 2: The global invariant set for the nominal dynamics of each topology $\Lambda$ can be computed as $\bar{\Omega}_{\Lambda}^{\mathrm{a}}=\bigotimes_{\mathcal{C} \in \mathcal{N} / \Lambda} \bar{\Omega}_{\mathcal{C}}^{\mathrm{a}}$. Also, notice that the nominal state of any coalition $\mathcal{C}$ lies in the projection of $\bar{\Omega}_{\mathcal{C}}^{\mathrm{a}}$ onto the axis corresponding to $\bar{x}_{\mathcal{C}}$. The equivalent conclusion holds for $\bar{x}_{\mathcal{N}}$ and $\bar{\Omega}_{\Lambda}^{\mathrm{a}}$.

\section{Objective function}

The objective function used in the computation of $\bar{u}_{\mathcal{C}}^{*}$ and $\bar{x}_{\mathcal{C}}^{*}$ is:

$$
\begin{aligned}
V_{\mathcal{C}}\left(\bar{x}_{\mathcal{C}}(0), \overline{\mathbf{u}}_{\mathcal{C}}, \bar{x}_{\mathcal{C}}^{\mathrm{s}}, \bar{u}_{\mathcal{C}}^{\mathrm{s}}, \bar{x}_{\mathcal{C}}^{\mathrm{t}}\right)= & \sum_{n=0}^{N_{\mathrm{p}}-1}\left(\left\|\bar{x}_{\mathcal{C}}(n)-\bar{x}_{\mathcal{C}}^{\mathrm{s}}\right\|_{Q_{\mathcal{C}}}^{2}+\left\|\bar{u}_{\mathcal{C}}(n)-\bar{u}_{\mathcal{C}}^{\mathrm{s}}\right\|_{R_{\mathcal{C}}}^{2}\right) \\
& +\left\|\bar{x}_{\mathcal{C}}\left(N_{\mathrm{p}}\right)-\bar{x}_{\mathcal{C}}^{\mathrm{s}}\right\|_{\bar{P}_{\mathcal{C}}}^{2}+V_{\mathrm{O}}^{\mathcal{C}}\left(\bar{x}_{\mathcal{C}}^{\mathrm{s}}, \bar{x}_{\mathcal{C}}^{\mathrm{t}}\right),
\end{aligned}
$$

where the time index between brackets indicates the step time of the prediction horizon, $Q_{\mathcal{C}}=\left(Q_{i}\right)_{i \in \mathcal{C}} \geq 0$ and $R_{\mathcal{C}}=$ $\left(R_{i}\right)_{i \in \mathcal{C}}>0$ are weighting matrices, with $Q_{i}$ and $R_{i}$ being the weighting matrices of agent $i, \overline{\mathbf{u}}_{\mathcal{C}}=\left[\bar{u}_{\mathcal{C}}(n)\right]_{n=0}^{N_{\mathrm{p}}-1}$ is the nominal sequence of inputs for a prediction horizon of $N_{\mathrm{p}}$ time steps, and $\left(\bar{x}_{\mathcal{C}}^{\mathrm{s}}, \bar{u}_{\mathcal{C}}^{\mathrm{s}}\right)$ is the setpoint, which becomes a variable that will be optimized each time instant. Note that this artificial setpoint remains constant during the prediction horizon. Additionally, $\left\|\bar{x}_{\mathcal{C}}\left(N_{\mathrm{p}}\right)-\bar{x}_{\mathcal{C}}^{\mathrm{s}}\right\| \frac{2}{\bar{P}_{\mathcal{C}}}$ is the terminal cost, being $\bar{P}_{\mathcal{C}}$ a properly chosen matrix, and $V_{\mathrm{O}}^{\mathcal{C}}(\cdot)$ is the offset cost function, which penalizes the deviation of $\bar{x}_{\mathcal{C}}^{\mathrm{s}}$ from the coalitional target set $\Gamma_{\mathcal{C}}$. In particular, we use

$$
V_{\mathrm{O}}^{\mathcal{C}}\left(\bar{x}_{\mathcal{C}}^{\mathrm{s}}, \bar{x}_{\mathcal{C}}^{\mathrm{t}}\right)=\alpha \sum_{i \in \mathcal{C}}\left\|\bar{x}_{i}^{\mathrm{s}}-\bar{x}_{i}^{\mathrm{t}}\right\|_{\infty},
$$

where $\alpha>0$ is a weighting parameter, and $\bar{x}_{\mathcal{C}}^{\mathrm{t}}=\left[\bar{x}_{i}^{\mathrm{t}}\right]_{i \in \mathcal{C}}$ is any point contained in the robust target set, i.e., $\psi_{\mathcal{C}}=\Gamma_{\mathcal{C}} \ominus \mathcal{R}_{\mathcal{C}}$.

Accordingly, the global objective function for any topology $\Lambda$ is defined by adding (14) for all $\mathcal{C} \in \mathcal{N} / \Lambda$, i.e.,

$V_{\mathcal{N}}\left(\bar{x}_{\mathcal{N}}(0), \overline{\mathbf{u}}_{\mathcal{N}}, \bar{x}_{\mathcal{N}}^{\mathrm{s}}, \bar{u}_{\mathcal{N}}^{\mathrm{s}}, \bar{x}_{\mathcal{N}}^{\mathrm{t}}, \Lambda\right)=\sum_{\mathcal{C} \in \mathcal{N} / \Lambda} V_{\mathcal{C}}\left(\bar{x}_{\mathcal{C}}(0), \overline{\mathbf{u}}_{\mathcal{C}}, \bar{x}_{\mathcal{C}}^{\mathrm{s}}, \bar{u}_{\mathcal{C}}^{\mathrm{s}}, \bar{x}_{\mathcal{C}}^{\mathrm{t}}\right)$, 
where $\overline{\mathbf{u}}_{\mathcal{N}}=\left[\overline{\mathbf{u}}_{\mathcal{C}}\right]_{\mathcal{C} \in \mathcal{N} / \Lambda}, \bar{x}_{\mathcal{N}}^{\mathrm{s}}=\left[\bar{x}_{\mathcal{C}}^{\mathrm{s}}\right]_{\mathcal{C} \in \mathcal{N} / \Lambda}, \bar{u}_{\mathcal{N}}^{\mathrm{s}}=\left[\bar{u}_{\mathcal{C}}^{\mathrm{s}}\right]_{\mathcal{C} \in \mathcal{N} / \Lambda}$ and $\bar{x}_{\mathcal{N}}^{\mathrm{t}}=\left[\bar{x}_{\mathcal{C}}^{\mathrm{t}}\right]_{\mathcal{C} \in \mathcal{N} / \Lambda}$. Note that $V_{\mathcal{N}}(\cdot)$ depends on $\Lambda$, i.e., the overall objective function changes with the topology.

Remark 3: The offset cost function (15) will be zero for all $\bar{x}_{\mathcal{C}}^{\mathrm{s}}$ contained in $\psi_{\mathcal{C}}$. As a consequence, any artificial steady state placed on the border of $\psi_{\mathcal{C}}$ cancels the offset cost.

\section{Optimization problem}

Every coalition solves the following problem at each time step:

$$
\begin{aligned}
\min _{\overline{\mathbf{u}}_{\mathcal{C}}, \bar{x}_{\mathcal{C}}(0), \bar{x}_{\mathcal{C}}^{\mathrm{s}}, \bar{u}_{\mathcal{C}}^{\mathrm{s}}, \bar{x}_{\mathcal{C}}^{\mathrm{t}}} & V_{\mathcal{C}}\left(\bar{x}_{\mathcal{C}}(0), \overline{\mathbf{u}}_{\mathcal{C}}, \bar{x}_{\mathcal{C}}^{\mathrm{s}}, \bar{u}_{\mathcal{C}}^{\mathrm{s}}, \bar{x}_{\mathcal{C}}^{\mathrm{t}}\right) \\
\text { s.t. } & \bar{x}_{\mathcal{C}}(0) \in x_{\mathcal{C}} \oplus\left(-\mathcal{R}_{\mathcal{C}}\right), \\
& \bar{x}_{\mathcal{C}}(n+1)=A_{\mathcal{C} \mathcal{x}} \bar{x}_{\mathcal{C}}(n)+B_{\mathcal{C}} \bar{u}_{\mathcal{C}}(n), \\
& \bar{u}_{\mathcal{C}}(n) \in \overline{\mathcal{U}}_{\mathcal{C}}=\mathcal{U}_{\mathcal{C}} \ominus K_{\mathcal{C}}^{\mathrm{e}} \mathcal{R}_{\mathcal{C}}, \\
& \bar{x}_{\mathcal{C}}(n) \in \overline{\mathcal{X}}_{\mathcal{C}}=\mathcal{X}_{\mathcal{C}} \ominus \mathcal{R}_{\mathcal{C}}, \\
& \bar{x}_{\mathcal{C}}^{\mathrm{t}} \in \psi_{\mathcal{C}}, \\
& \left(\bar{x}_{\mathcal{C}}\left(N_{\mathrm{p}}\right), \bar{x}_{\mathcal{C}}^{\mathrm{s}}, \bar{u}_{\mathcal{C}}^{\mathrm{s}}\right) \in \bar{\Omega}_{\mathcal{C}}^{\mathrm{a}}, \\
& \forall n=0, \ldots, N_{\mathrm{p}}-1,
\end{aligned}
$$

where the state and input constraints are shrunk to account for the possible future realizations of the disturbances. The minimizer of (17) is formed by the optimal coalitional sequence of inputs $\overline{\mathbf{u}}_{\mathcal{C}}^{*}$ and initial state $\bar{x}_{\mathcal{C}}^{*}(0)$, together with the optimal artificial states and inputs $\left(\bar{x}_{\mathcal{C}}^{\mathrm{s} *}, \bar{u}_{\mathcal{C}}^{\mathrm{s} *}\right)$, and a certain $\bar{x}_{\mathcal{C}}^{\mathrm{t} *}$. Note that if $\Lambda_{\mathrm{dec}}$ is imposed, then each agent considers only its local objective and variables, thus minimizing the communication burden and the local problems complexity. Conversely, if $\Lambda_{\text {cen }}$ is used, then all agents jointly optimize the overall objective, thus maximizing the global performance.

Remark 4: Let the system be controlled using a static topology $\Lambda$ and let $u_{\mathcal{C}}=\bar{u}_{\mathcal{C}}^{*}+K_{\mathcal{C}}^{\mathrm{e}}\left(x_{\mathcal{C}}-\bar{x}_{\mathcal{C}}^{*}\right)$ be the control law of any cluster $\mathcal{C} \in \mathcal{N} / \Lambda$, where $\bar{u}_{\mathcal{C}}^{*}$, and $\bar{x}_{\mathcal{C}}^{*}$ are respectively the first component of $\overline{\mathbf{u}}_{\mathcal{C}}^{*}$ and the initial state obtained from (17). Then, the global state trajectory will evolve in the tube defined by $\mathcal{R}_{\Lambda}=\otimes_{\mathcal{C} \in \mathcal{N} / \Lambda} \mathcal{R}_{\mathcal{C}}$ [31, Proposition 1], i.e., the deviation between the predicted and real trajectories is bounded.

\section{E. Topology Switching Features}

We consider a double sample rate strategy where every $T_{\text {top }}$ steps a network topology is selected through a comparative assessment of different configurations.

1) Topologies evaluation: The following index is used as a surrogate to evaluate all $\Lambda \in \mathcal{T}$ [8]:

$$
r\left(\Lambda, x_{\mathcal{N}}\right)=\left(x_{\mathcal{N}}-x_{\Gamma}\right)^{\mathrm{T}} P_{\Lambda}\left(x_{\mathcal{N}}-x_{\Gamma}\right)+c|\Lambda|,
$$

where $x_{\Gamma}$ is the Chebyshev center of the target set, $c>0$ is the cost of enabling a link and $|\Lambda|$ is the number of enabled links in $\Lambda$. The first term of index (18) provides an upper bound estimate of the unconstrained performance costs associated with topology $\Lambda$, while the second term weights the coordination costs and promotes the use of sparse topologies. The procedure to obtain $P_{\Lambda}$ is detailed in [8].
2) Condition on the switchings: A change of topology entails a switching of both the nominal control model and the state and input constraints in (17). In particular, notice that splitting any coalition increases the uncertainty of the corresponding subsystems, leading to greater reductions of the constraints sets in (17). For stability and feasibility purposes, we impose two conditions on the switchings:

(i) A transition to $\Lambda^{+}$takes place iff the system state at the switching instant fulfills $x_{\mathcal{N}} \in \operatorname{Proj}_{x} \bar{\Omega}_{\Lambda^{+}}^{\mathrm{a}}$, where $\operatorname{Proj}_{x} \bar{\Omega}_{\Lambda^{+}}^{\mathrm{a}}$ is the projection of the augmented invariant set of topology $\Lambda^{+}$onto $x_{\mathcal{N}}$.

(ii) The topologies remain active for a period that guarantees a decreasing evolution of nominal costs (16).

Note that if sets $\operatorname{Proj}_{x} \bar{\Omega}_{\Lambda^{+}}^{\mathrm{a}}$ are computed offline for all possible $\Lambda^{+}$, then (i) translates into checking if a matrix inequality is satisfied. Alternatively, it can be checked if there exists some $\left(\bar{x}_{\mathcal{N}}^{\mathrm{s}}, \bar{u}_{\mathcal{N}}^{\mathrm{s}}\right)$ such that $\left(x_{\mathcal{N}}, \bar{x}_{\mathcal{N}}^{\mathrm{s}}, \bar{u}_{\mathcal{N}}^{\mathrm{s}}\right) \in \bar{\Omega}_{\Lambda^{+}}^{\mathrm{a}}$. The latter can be formulated as a simple optimization that would be solved every $T_{\text {top }}$ instants with minimum computation burden.

\section{Control Scheme}

The robust coalitional control scheme for tracking target sets introduced in this work is presented next:

Control Scheme 1: Let us consider that the communication topologies may be switched every $T_{\text {top }}$ time instants with the set $\mathcal{K}=\left\{p T_{\text {top }}: p \in \mathbb{N}_{>0}\right\}$ containing the switching instants. Also, set the topology $\Lambda$ to an initial configuration, say $\Lambda_{\text {ini }}$, and the initial value of $\bar{V}$ to infinity. Then, at each sample time $k$ proceed as follows:

1: if $k \in \mathcal{K}$ then

2: Coalitions share their state and current costs.

3: $\quad$ if $V_{\mathcal{N}}^{*}<\bar{V}$ then

4: $\quad$ Topology $\Lambda^{+} \in \mathcal{T}$ that minimizes (18) is selected.

5: $\quad$ If $x_{\mathcal{N}} \in \operatorname{Proj}_{x} \bar{\Omega}_{\Lambda^{+}}^{\mathrm{a}}$, update $\bar{V}=V_{\mathcal{N}^{*}}$ and set $\Lambda=\Lambda^{+}$.

6: $\quad$ end if

7: end if

8: Each cluster $\mathcal{C} \in \mathcal{N} / \Lambda$ computes $\overline{\mathbf{u}}_{\mathcal{C}}^{*}$ and $\bar{x}_{\mathcal{C}}^{*}$ through (17).

9: Each cluster $\mathcal{C} \in \mathcal{N} / \Lambda$ implements tube-based control law $u_{\mathcal{C}}=\bar{u}_{\mathcal{C}}^{*}+K_{\mathcal{C}}^{\mathrm{e}}\left(x_{\mathcal{C}}-\bar{x}_{\mathcal{C}}^{*}\right)$, where $\bar{u}_{\mathcal{C}}^{*}$, and $\bar{x}_{\mathcal{C}}^{*}$ are obtained from (17).

Note that Control Scheme 1 uses the same tracking methodology as the distributed MPC in [27], which is also extended in [28] by exploiting the subsystems interactions to reduce the communication overhead. However, there are notable differences, among which are the following: in [27], [28], all agents are cooperative and all (interactive) subsystems communicate to optimize a plant-wide objective, while here each cluster optimizes the aggregate benefit of its members and there is no inter-coalition communication; [27], [28] guarantees convergence to the centralized optimum, while the coalitional controller balances the coordination effort and system performance; also, [27] is based on a warm-start initialization that is not used in this article.

Remark 5: Set $\mathcal{T}$ in Control Scheme 1 may not contain all possible topologies and may be adapted in real time using a reasonably-sized subset of candidate topologies [12]. Also, notice that the minimum and maximum topologies in $\mathcal{T}$ are 
not necessarily $\Lambda_{\text {dec }}$ and $\Lambda_{\text {cen }}$. Hence, $\Lambda_{\text {cen }}$ may be replaced by some other configuration involving smaller clusters, thus simplifying the computations at expense of a certain loss of performance. Similarly, if the coupling conditions are such that Assumptions 1 or 3 do not hold for $\Lambda_{\mathrm{dec}}$, then other topology can be used as sparsest viable configuration. Finally, note that it is possible to check offline if some topology involves clusters that do not satisfy these assumptions, and hence to remove it from the set of configurations that may be chosen.

Remark 6: By using homothetic, elastic and polytopic tube-based approaches [36]-[38], conservatism in Control Scheme 1 can be reduced. Another possibility is introduced in [39], where the worst-case constraint tightening, as used in (17), is replaced by an optimization-based strategy where these constraints reduction depends on decision variables. Similarly, [40] proposes a tube-based DMPC where agents share data so as to reduce the size of the coupling uncertainty.

Let $\mathcal{S}_{N_{\mathrm{p}}}$ be the domain of attraction of the initial topology $\Lambda_{\text {ini }}$. Then, the following two theorems hold:

Theorem 1 (Recursive feasibility): For any initial state $x_{\mathcal{N}} \in$ $\mathcal{S}_{N_{\mathrm{p}}}$, feasibility is guaranteed along the closed-loop trajectory of the system.

Proof 1: Let us first consider that the topology remains fixed. In this case, recursive feasibility is proven below following [29]. In particular, consider instant $k$, let $\Lambda$ be the topology, and let $\left(\bar{x}_{\mathcal{C}}^{*}(0), \overline{\mathbf{u}}_{\mathcal{C}}^{*}, \bar{x}_{\mathcal{C}}^{\mathrm{s*}}, \bar{u}_{\mathcal{C}}^{\mathrm{s} *}, \bar{x}_{\mathcal{C}}^{\mathrm{t} *}\right)$ be the solution of (17) for cluster $\mathcal{C} \in \mathcal{N} / \Lambda$, with $\overline{\mathbf{x}}_{\mathcal{C}}^{*}=\left[\bar{x}_{\mathcal{C}}^{*}(n)\right]_{n=0}^{N_{\mathrm{p}}-1}$ being the corresponding sequence of predicted states. Additionally, take

$$
\begin{aligned}
& \tilde{\mathbf{u}}_{\mathcal{C}}^{+}=\left[\begin{array}{c}
\tilde{u}_{\mathcal{C}}^{+}(0) \\
\vdots \\
\tilde{u}_{\mathcal{C}}^{+}\left(N_{\mathrm{p}}-2\right) \\
\tilde{u}_{\mathcal{C}}^{+}\left(N_{\mathrm{p}}-1\right)
\end{array}\right]=\left[\begin{array}{c}
\bar{u}_{\mathcal{C}}^{*}(1) \\
\vdots \\
\bar{u}_{\mathcal{C}}^{*}\left(N_{\mathrm{p}}-1\right) \\
\bar{u}_{\mathcal{C}}+\bar{K}_{\mathcal{C}}\left(\bar{x}_{\mathcal{C}}^{*}\left(N_{\mathrm{p}}\right)-\bar{x}_{\mathcal{C}}^{\mathrm{s} *}\right)
\end{array}\right], \\
& \tilde{x}_{\mathcal{C}}^{+}(0)=\bar{x}_{\mathcal{C}}^{*}(1), \quad \tilde{x}_{\mathcal{C}}^{\mathrm{s}+}=\bar{x}_{\mathcal{C}}^{\mathrm{s*}}, \quad \tilde{u}_{\mathcal{C}}^{\mathrm{s}+}=\bar{u}_{\mathcal{C}}^{\mathrm{s} *}, \quad \tilde{x}_{\mathcal{C}}^{\mathrm{t}}=\bar{x}_{\mathcal{C}}^{\mathrm{t}},
\end{aligned}
$$

as a candidate solution for instant $k+1$. Then, the following holds:

(i) From the notion of tubes [31], the coalition state at $k+1$ is such that $x_{\mathcal{C}}^{+} \in \bar{x}_{\mathcal{C}}^{*}(1) \oplus \mathcal{R}_{\mathcal{C}}$, and hence $x_{\mathcal{C}}^{+} \in \tilde{x}_{\mathcal{C}}^{+}(0) \oplus \mathcal{R}_{\mathcal{C}}$.

(ii) Admissibility of $\overline{\mathbf{u}}_{\mathcal{C}}^{*}$ implies that inputs $\tilde{u}_{\mathcal{C}}^{+}\left(0: N_{\mathrm{p}}-2\right)=$ $\bar{u}_{\mathcal{C}}^{*}\left(1: N_{\mathrm{p}}-1\right)$ are admissible.

(iii) Variable $\tilde{x}_{\mathcal{C}}^{+}\left(N_{\mathrm{p}}-1\right)$, i.e., the predicted nominal state at $k+1$ for instant $k+N_{\mathrm{p}}$, is given by $\bar{x}_{\mathcal{C}}^{*}\left(N_{\mathrm{p}}\right)$, hence,

$$
\left(\tilde{x}_{\mathcal{C}}^{+}\left(N_{\mathrm{p}}-1\right), \tilde{x}_{\mathcal{C}}^{\mathrm{s}+}, \tilde{u}_{\mathcal{C}}^{\mathrm{s}+}\right) \in \bar{\Omega}_{\mathcal{C}}^{\mathrm{a}},
$$

and, therefore, $\tilde{u}_{\mathcal{C}}^{+}\left(N_{\mathrm{p}}-1\right)=\bar{u}_{\mathcal{C}}^{\mathrm{s} *}+\bar{K}_{\mathcal{C}}\left(\tilde{x}_{\mathcal{C}}^{+}\left(N_{\mathrm{p}}-1\right)-\bar{x}_{\mathcal{C}}^{\mathrm{s} *}\right)$ is also admissible.

(iv) Finally, the invariance of $\bar{\Omega}_{\mathcal{C}}^{\mathrm{a}}$ entails

$$
\left(\tilde{x}_{\mathcal{C}}^{+}\left(N_{\mathrm{p}}\right), \tilde{x}_{\mathcal{C}}^{\mathrm{s}+}, \tilde{u}_{\mathcal{C}}^{\mathrm{s}+}\right) \in \bar{\Omega}_{\mathcal{C}}^{\mathrm{a}} .
$$

Therefore, for all $\mathcal{C} \in \mathcal{N} / \Lambda,\left(\tilde{x}_{\mathcal{C}}^{+}(0), \tilde{\mathbf{u}}_{\mathcal{C}}^{+}, \tilde{x}_{\mathcal{C}}^{\mathrm{s}+}, \tilde{u}_{\mathcal{C}}^{\mathrm{s}+}, \tilde{x}_{\mathcal{C}}^{\mathrm{t}+}\right)$ constitutes a feasible solution of (17) for instant $k+1$. Recursive feasibility for any static $\Lambda$ follows by induction.

On the other hand, the condition imposed on the switchings prevents unfeasibility problems when the clusters are changed. The latter is the only condition that needs to be checked online to guarantee feasibility. In particular, a transition to a new topology $\Lambda^{+}$takes place iff the overall system state $x_{\mathcal{N}}$ is such that $x_{\mathcal{N}} \in \operatorname{Proj}_{x} \bar{\Omega}_{\Lambda^{+}}^{\mathrm{a}}$, i.e., $x_{\mathcal{C}} \in \operatorname{Proj}_{x} \bar{\Omega}_{\mathcal{C}}^{\mathrm{a}}$, for all $\mathcal{C} \in \mathcal{N} / \Lambda^{+}$. Hence, there exists at least one feasible artificial steady state and input $\left(\hat{x}_{\mathcal{C}}^{\mathrm{s}+}, \hat{u}_{\mathcal{C}}^{\mathrm{s}+}\right)$ such that

$$
\left(x_{\mathcal{C}}, \hat{x}_{\mathcal{C}}^{\mathrm{s}+}, \hat{u}_{\mathcal{C}}^{\mathrm{s}+}\right) \in \bar{\Omega}_{\mathcal{C}}^{\mathrm{a}}, \quad \forall \mathcal{C} \in \mathcal{N} / \Lambda^{+} .
$$

Additionally, feasible inputs can be derived from (11), i.e.,

$$
\hat{\mathbf{u}}_{\mathcal{C}}^{+}=\left[\hat{u}_{\mathcal{C}}^{+}(n)\right]_{n=0}^{N_{\mathrm{p}}-1}=\left[\hat{u}_{\mathcal{C}}^{\mathrm{s}+}+\bar{K}_{\mathcal{C}}\left(\bar{x}_{\mathcal{C}}(n)-\hat{x}_{\mathcal{C}}^{\mathrm{s}+}\right)\right]_{n=0}^{N_{\mathrm{p}}-1},
$$

where $\bar{x}_{\mathcal{C}}(n+1)=A_{\mathcal{C C}} \bar{x}_{\mathcal{C}}(n)+B_{\mathcal{C C}} \hat{u}_{\mathcal{C}}(n)$ and $\bar{x}_{\mathcal{C}}(0)=$ $x_{\mathcal{C}}$. Therefore, the existence of a feasible initial state, a feasible sequence of inputs, and a feasible equilibrium point $\left(x_{\mathcal{C}}, \hat{\mathbf{u}}_{\mathcal{C}}^{+}, \hat{x}_{\mathcal{C}}^{\mathrm{s}}, \hat{u}_{\mathcal{C}}^{\mathrm{s}+}\right)$ is guaranteed. Finally, note that variable $\bar{x}_{\mathcal{C}}^{\mathrm{t}}$ cannot itself make problem (17) unfeasible.

Considering the above and that the initial topology is $\Lambda_{\mathrm{ini}}$, recursive feasibility is guaranteed for all initial states within $\mathcal{S}_{N_{\mathrm{p}}}$.

Theorem 2 (Stability): For any initial state $x_{\mathcal{N}} \in \mathcal{S}_{N_{\mathrm{p}}}$, asymptotic stability is guaranteed.

Proof 2: Firstly, we will prove that function (16) is a decreasing function when the network topology is static. Similarly, consider time instant $k$, let $\Lambda$ be the network topology, and let $\left(\bar{x}_{\mathcal{C}}^{*}(0), \overline{\mathbf{u}}_{\mathcal{C}}^{*}, \bar{x}_{\mathcal{C}}^{\mathrm{s} *}, \bar{u}_{\mathcal{C}}^{\mathrm{s} *}, \bar{x}_{\mathcal{C}}^{\mathrm{t} *}\right)$ be the minimizer of (17) for cluster $\mathcal{C} \in \mathcal{N} / \Lambda$. Then,

$$
V_{\mathcal{C}}^{*}=V_{\mathcal{C}}\left(\bar{x}_{\mathcal{C}}^{*}(0), \overline{\mathbf{u}}_{\mathcal{C}}^{*}, \bar{x}_{\mathcal{C}}^{\mathrm{s} *}, \bar{u}_{\mathcal{C}}^{\mathrm{s} *}, \bar{x}_{\mathcal{C}}^{\mathrm{t} *}\right),
$$

is its optimal (nominal) performance cost attained at $k$. Let us move one step forward and consider candidate solution (19). By optimality, $\tilde{V}_{\mathcal{C}}^{+}=V_{\mathcal{C}}\left(\tilde{x}_{\mathcal{C}}^{+}(0), \tilde{\mathbf{u}}_{\mathcal{C}}^{+}, \tilde{x}_{\mathcal{C}}^{\mathrm{s}+}, \tilde{u}_{\mathcal{C}}^{\mathrm{s}+}, \tilde{x}_{\mathcal{C}}^{\mathrm{t}+}\right)$ provides an upper bound on the optimal cost at time instant $k+1$. In particular, we can write:

$$
\begin{aligned}
\tilde{V}_{\mathcal{C}}^{+} & =V_{\mathcal{C}}^{*}-\left\|\bar{x}_{\mathcal{C}}-\bar{x}_{\mathcal{C}}^{\mathrm{s} *}\right\|_{Q_{\mathcal{C}}}^{2}-\left\|\bar{u}_{\mathcal{C}}^{*}(0)-\bar{u}_{\mathcal{C}}^{\mathrm{s} *}\right\|_{R_{\mathcal{C}}}^{2} \\
& +\left\|\tilde{x}_{\mathcal{C}}^{+}\left(N_{\mathrm{p}}-1\right)-\tilde{x}_{\mathcal{C}}^{\mathrm{s}+}\right\|_{Q_{\mathcal{C}}}^{2}+\left\|\tilde{u}_{\mathcal{C}}^{+}\left(N_{\mathrm{p}}-1\right)-\tilde{u}_{\mathcal{C}}^{\mathrm{s}+}\right\|_{R_{\mathcal{C}}}^{2} \\
& -\left\|\bar{x}_{\mathcal{C}}^{*}\left(N_{\mathrm{p}}\right)-\bar{x}_{\mathcal{C}}^{\mathrm{s} *}\right\|_{P_{\mathcal{C}}}^{2}+\left\|\tilde{x}_{\mathcal{C}}^{+}\left(N_{\mathrm{p}}\right)-\tilde{x}_{\mathcal{C}}^{\mathrm{s}+}\right\|_{P_{\mathcal{C}}}^{2},
\end{aligned}
$$

where we have considered that the offset cost remains constant since $\bar{x}_{\mathcal{C}}^{\mathrm{s} *}=\tilde{x}_{\mathcal{C}}^{\mathrm{s}+}$ and $\bar{x}_{\mathcal{C}}^{\mathrm{t*}}=\tilde{x}_{\mathcal{C}}^{\mathrm{t}+}$. Also, the terminal cost satisfies

$$
\begin{gathered}
\left\|\tilde{x}_{\mathcal{C}}^{+}\left(N_{\mathrm{p}}-1\right)-\tilde{x}_{\mathcal{C}}^{\mathrm{s}+}\right\|_{Q_{\mathcal{C}}}^{2}+\left\|\tilde{u}_{\mathcal{C}}^{+}\left(N_{\mathrm{p}}-1\right)-\tilde{u}_{\mathcal{C}}^{\mathrm{s}+}\right\|_{R_{\mathcal{C}}}^{2} \\
-\left\|\bar{x}_{\mathcal{C}}^{*}\left(N_{\mathrm{p}}\right)-\bar{x}_{\mathcal{C}}^{\mathrm{s} *}\right\|_{P_{\mathcal{C}}}^{2}+\left\|\tilde{x}_{\mathcal{C}}^{+}\left(N_{\mathrm{p}}\right)-\tilde{x}_{\mathcal{C}}^{\mathrm{s}+}\right\|_{P_{\mathcal{C}}}^{2} \leq 0 .
\end{gathered}
$$

Then, $\tilde{V}_{\mathcal{C}}^{+}-V_{\mathcal{C}}^{*} \leq-\left\|\bar{x}_{\mathcal{C}}-\bar{x}_{\mathcal{C}}^{\mathrm{s} *}\right\|_{Q_{\mathcal{C}}}^{2}-\left\|\bar{u}_{\mathcal{C}}^{*}(0)-\bar{u}_{\mathcal{C}}^{\mathrm{s} *}\right\|_{R_{\mathcal{C}}}^{2}$, and, hence,

$$
V_{\mathcal{C}}^{*+}-V_{\mathcal{C}}^{*} \leq-\left\|\bar{x}_{\mathcal{C}}-\bar{x}_{\mathcal{C}}^{\mathrm{s}}\right\|_{Q_{\mathcal{C}}}^{2} .
$$

Since (27) holds for all clusters $\mathcal{C} \in \mathcal{N} / \Lambda$, the overall optimal (nominal) costs, i.e., $V_{\mathcal{N}}^{*}=\sum_{\mathcal{C} \in \mathcal{N} / \Lambda} V_{\mathcal{C}}^{*}$, satisfy

$$
V_{\mathcal{N}}^{*+}-V_{\mathcal{N}}^{*} \leq-\left\|\bar{x}_{\mathcal{N}}-\bar{x}_{\mathcal{N}^{\mathrm{s}}}^{*}\right\|_{Q_{\mathcal{N}}}^{2} .
$$

From (28), we have that the overall nominal state tends to the artificial steady state as time goes to infinity, and following similar arguments to Lemma 1 in [29], it can be proven that the artificial steady state converges to a fixed point, for any static network topology. On the other hand, the switchings of topologies, and hence the clusters modifications, may cause temporary increases of the optimal nominal costs. That is, condition (28) does not hold generally for all time steps. However, Control Scheme 1 guarantees that function $V_{\mathcal{N}}^{*}$ sampled at the instants prior to the switchings always decreases with 
time, which assures asymptotic stability of the overall nominal switching system.

From the theorems above, we can deduce that as time goes to infinity, function $V_{\mathcal{N}}^{*}$ tends to a constant. Since the topology switchings are subject to a decrease of $V_{\mathcal{N}^{*}}^{*}$, from a certain time instant the network configuration will remain static with some $\Lambda_{\infty}$. In this regard, given (28), the nominal system state at infinity, say $\bar{x}_{\mathcal{N}, \infty}$, converges to steady state $\bar{x}_{\mathcal{N}, \infty}^{\mathrm{s} *}$. Additionally, from the notion of tubes, it is guaranteed that the real state converges to a neighbourhood of the nominal system defined by $\mathcal{R}_{\Lambda_{\infty}}$, i.e., $x_{\mathcal{N}, \infty} \in \bar{x}_{\mathcal{N}, \infty}^{\mathrm{s} *} \oplus \mathcal{R}_{\Lambda_{\infty}}$. Previous works on MPC for tracking [26], [29] prove that this final operating point $\bar{x}_{\mathcal{N}, \infty}^{\mathrm{s} *}$ is defined as a minimizer of the offset cost function subject to the constraints in (17). In particular, let us define $\overline{\mathcal{X}}_{\mathcal{C}}^{\mathrm{s}}=\left\{\bar{x}_{\mathcal{C}}^{\mathrm{s}}:\left(\bar{x}_{\mathcal{C}}^{\mathrm{s}}, \bar{x}_{\mathcal{C}}^{\mathrm{s}}, \bar{u}_{\mathcal{C}}^{\mathrm{s}}\right) \in \bar{\Omega}_{\mathcal{C}}^{\mathrm{a}}\right\}$ as the set of admissible steady states consistent with the invariant set for tracking of cluster $\mathcal{C}$. Under the assumption that $\psi_{\mathcal{C}} \cap \overline{\mathcal{X}}_{\mathcal{C}}^{\mathrm{s}} \neq \emptyset$ for all $\mathcal{C} \in \mathcal{N} / \Lambda_{\infty}$, it is verified that $\bar{x}_{\mathcal{C}, \infty}^{\mathrm{s} *} \in \psi_{\mathcal{C}}$ for all $\mathcal{C} \in \mathcal{N} / \Lambda_{\infty}$. Hence, from a global viewpoint, the following holds:

$$
x_{\mathcal{N}, \infty} \in \bar{x}_{\mathcal{N}, \infty}^{\mathrm{s} *}+\mathcal{R}_{\Lambda_{\infty}} \subseteq \psi_{\mathcal{N}} \oplus \mathcal{R}_{\Lambda_{\infty}} \subseteq \Gamma_{\mathcal{N}}
$$

where $\psi_{\mathcal{N}}=\otimes_{\mathcal{C} \in \mathcal{N} / \Lambda_{\infty}} \psi_{\mathcal{C}}$.

\section{Simulation Results}

In this section, we apply the coalitional scheme to a modified version of the system proposed in [40], [41]. It consists of a set $\mathcal{N}=\{1,2, \ldots, 12\}$ of trucks coupled via springs and dampers as shown in Fig. 2, with dynamics modeled by

$$
\left[\begin{array}{c}
\dot{s}_{i} \\
\dot{v}_{i}
\end{array}\right]=A_{i i}\left[\begin{array}{c}
s_{i} \\
v_{i}
\end{array}\right]+B_{i i} u_{i}+\sum_{j \in \mathcal{N}_{i}} A_{i j}\left[\begin{array}{c}
s_{j} \\
v_{j}
\end{array}\right]+d_{i},
$$

where

$$
\begin{aligned}
& A_{i i}=\left[\begin{array}{cc}
0 & 1 \\
-\frac{1}{m_{i}} \sum_{j \in \mathcal{N}_{i}} k_{i j} & -\frac{1}{m_{i}} \sum_{j \in \mathcal{N}_{i}} h_{i j}
\end{array}\right], B_{i i}=\left[\begin{array}{c}
0 \\
50
\end{array}\right], \\
& A_{i j}=\left[\begin{array}{cc}
0 & 0 \\
\frac{1}{m_{i}} \sum_{j \in \mathcal{N}_{i}} k_{i j} & \frac{1}{m_{i}} \sum_{j \in \mathcal{N}_{i}} h_{i j}
\end{array}\right], \quad i \neq j .
\end{aligned}
$$

The state $x_{i}$ of each subsystem is formed by its displacement $s_{i}$ from the equilibrium point and its instantaneous velocity $v_{i}$. Additionally, the agents can apply a longitudinal

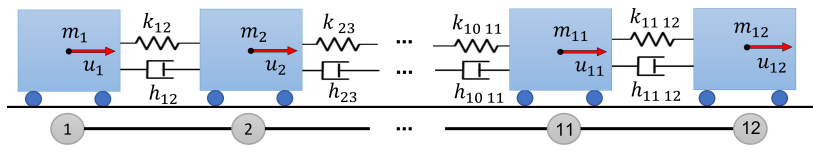

\begin{tabular}{|c|c|c|c|c|c|}
\hline $\begin{array}{l}k_{12}, k_{45}, \\
k_{78}, k_{1011}\end{array}$ & 0.4 & $\begin{array}{l}k_{23}, k_{56} \\
k_{89}, k_{1112}\end{array}$ & 0.8 & $\begin{array}{l}k_{34}, k_{67}, \\
k_{910}\end{array}$ & 0.9 \\
\hline $\begin{array}{l}h_{12}, h_{67}, \\
h_{910}\end{array}$ & 0.2 & $\begin{array}{l}h_{23}, h_{45}, \\
h_{78}, h_{1011}\end{array}$ & 0.1 & $\begin{array}{l}h_{34}, h_{56}, \\
h_{89}, h_{1112}\end{array}$ & 0.15 \\
\hline \multicolumn{3}{|c|}{ Masses [kg] } & \multicolumn{3}{|c|}{ Other parameters } \\
\hline $\begin{array}{l}m_{1}, m_{6}, \\
m_{2}, m_{4},\end{array}$ & $\begin{array}{l}n_{12} \\
n_{10}\end{array}$ & $\begin{array}{l}3 \\
4\end{array}$ & 0. & \begin{tabular}{l|l} 
& $T_{\text {top }}$
\end{tabular} & $0.8 \mathrm{~s}$ \\
\hline$m_{3}, m_{5}$ & & 5 & & & \\
\hline
\end{tabular}

Fig. 2: 12-truck system and related communication network.

TABLE I: Parameters used in the simulation Spring stiffnesses $[\mathrm{N} / \mathrm{m}]$ and damping factors $[\mathrm{N} /(\mathrm{m} \times \mathrm{s})]$

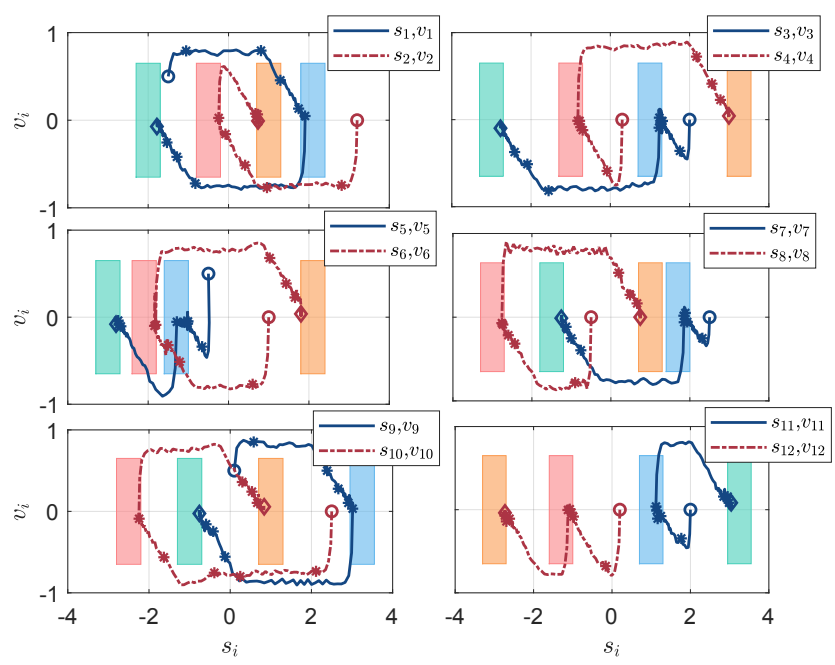

Fig. 3: Evolution of the system to two target sets. The subsystems states at the switching instants are indicated by star marks, and the initial and final states are marked respectively with a circle and a diamond. The light blue and red regions represent the first target sets for the corresponding subsystems, and green and orange regions the second ones.

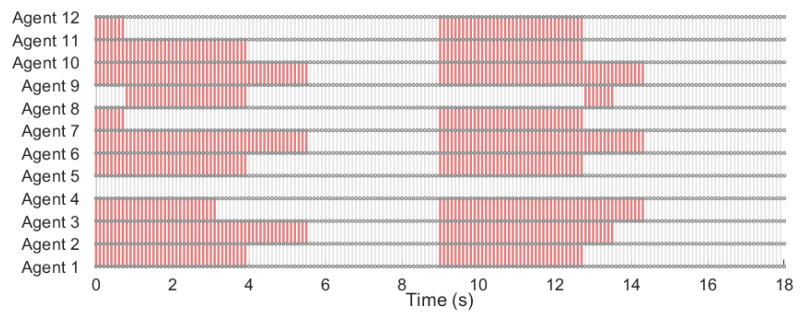

Fig. 4: Topology evolution over time. It can be seen how the links are progressively disabled as the trucks approach the target sets.

force $F_{i}=50 u_{i} \mathrm{~N}$, where $u_{i}$ is the control action. The parameters that characterize the system are given in Table I. The continuous-time dynamics are discretized using zero-order hold and a sampling time of $0.1 \mathrm{~s}$. The goal is to regulate the four trucks towards a target set while allowing switchings on the network topology and satisfying $\left|s_{i}\right| \leq 4$ and $\left|u_{i}\right| \leq 1$. In this regard, the agents are connected by a network of 11 links as shown in Fig. 2. In addition, we have considered an external disturbance, which is defined as a bounded white noise as $[-0.003,-0.03]^{\mathrm{T}} \leq d_{i} \leq[0.003,-0.03]^{\mathrm{T}}$, for all $i \in \mathcal{N}$. Finally, the stage cost is defined by weighting

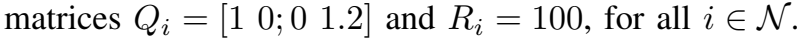

For the design of gains $K_{\mathcal{C}}^{\mathrm{e}}$ and matrices $P_{\Lambda}$ we have followed [29] and [8], respectively. To this end, we have used the Matlab ${ }^{\circledR}$ LMI Control Toolbox [42] and specifically the solver mincx, in a $1.8 \mathrm{GHz}$ quad-core Intel $^{\circledR}$ Core $^{\mathrm{TM}}$ i7/8 GB RAM computer. On the other hand, feedback gains $\bar{K}_{\mathcal{C}}$ and matrices $\bar{P}_{\mathcal{C}}$ have been calculated using function dlqr from Matlab ${ }^{\circledR}$. Sets $\mathcal{R}_{\Lambda}$ are designed following [43], where a one-step approach based on a linear program is proposed for computing minimal robust positively invariant sets. Finally, the augmented invariant sets for tracking have been computed using the Multi-Parametric Toolbox 3.0 [44], where for converge reasons a factor $\lambda=0.97$ has 
been used (see [24]). Due to the high computational burden of operating with high-dimensional sets, we have limited the coalitions size to $|\mathcal{C}| \leq 4$. The latter pre-selects a subset of 1450 topologies among the $2^{11}=2048$ combinations of enabled and disabled links, and allows to manage the system without requiring great efforts to compute the constraints sets of (17) for any coalition, at expense of limiting the maximum degree of coordination that can be attained. Nevertheless, notice that using a zonotope representation of the polytopes involved in (17) can facilitate the constraints sets computation for larger clusters. Likewise, the methodology in [29], which scales poorly for high dimensional disturbance sets, can be replaced by any other method providing matrices $K_{\mathcal{C}}^{\mathrm{e}}$ satisfying Assumption 1. The results obtained from the simulation of Control Scheme 1 with the parameters in Table I and using as initial topology $\Lambda_{\text {ini }}$ the one shown in Fig. 4 are summarized below.

Fig. 3 illustrates the subsystems' state trajectories on planes $\left(s_{i}, v_{i}\right)$ with the coalitional controller, and Fig. 4 shows the network topologies used over time. The simulation lasts for $18 \mathrm{~s}$, with a change of target set at $9 \mathrm{~s}$. When the target is changed, topology $\Lambda_{\text {ini }}$ is imposed and $\bar{V}$ is set to $\infty$. As shown in Fig. 4, when the system is in the vicinity of the target, a decentralized control suffices to drive and maintain the overall state within this region. The use of topologies with one or more enabled links reduces the coupling uncertainty of the merged agents in comparison to a static decentralized configuration, which leads to less restrictive constraints during part of the simulation (see the projections of sets $\overline{\mathcal{X}}_{\Lambda_{\mathrm{dec}}}$ and $\overline{\mathcal{X}}_{\Lambda_{\text {ini }}}$ onto plane $\left(s_{9}, v_{9}\right)$ in Fig. 5). Note that the decentralized controller refers to the case in which $\Lambda_{\mathrm{dec}}$ is permanently imposed, that is, each local agent performs as the robust MPC for tracking introduced in [29], but considering that the disturbances account for both coupling effect and external perturbations. For the sake of comparison, we show the results obtained with the centralized MPC in [26] with perfect external disturbances forecast and without shrinking constraint sets $\mathcal{X}_{\mathcal{N}}$ and $\mathcal{U}_{\mathcal{N}}$. Note that the latter provides a lower bound on the performance costs that can be attained and uses the centralized model (2) derived from (30) as prediction model.

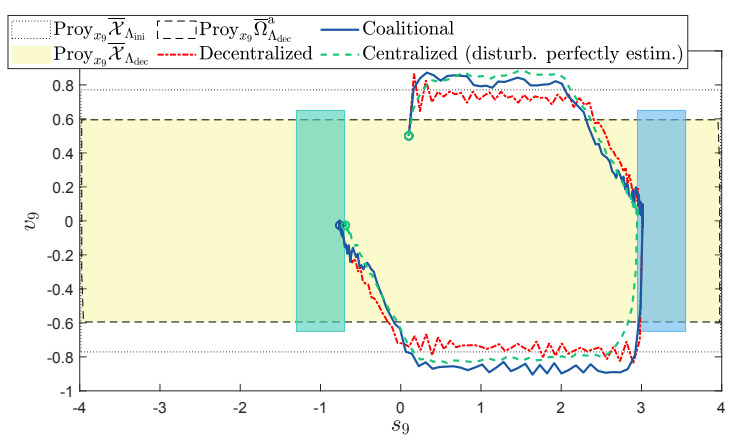

Fig. 5: Trajectory of truck 9 on plane $\left(s_{9}, v_{9}\right)$ when using the decentralized, coalitional and centralized controllers. The figure also shows how the coupling uncertainty reduces the decentralized topology constraint set $\overline{\mathcal{X}}_{\Lambda_{\mathrm{dec}}}$ in comparison with $\overline{\mathcal{X}}_{\Lambda_{\mathrm{ini}}}$. Additionally, it shows that the projection of the augmented invariant set for tracking $\bar{\Omega}_{\Lambda_{\mathrm{dec}}}^{\mathrm{a}}$ contains practically all points in $\overline{\mathcal{X}}_{\Lambda_{\mathrm{dec}}}$.

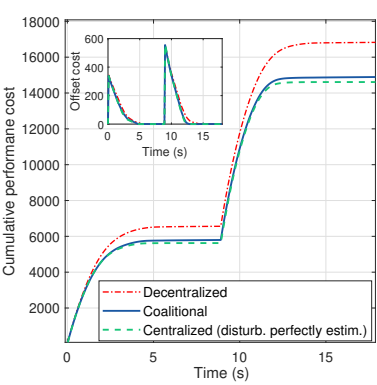

(a) Performance cost.

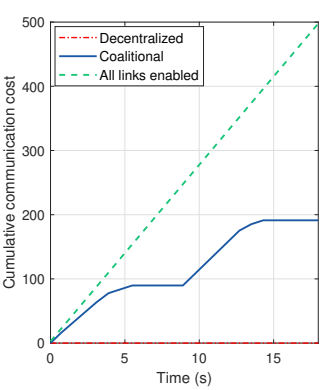

(b) Communication costs.
Fig. 6: Comparison between the centralized, coalitional and decentralized cumulative costs. In (a), the evolution of the offset cost is also shown, i.e., the value of $\alpha \sum_{i \in \mathcal{N}}\left\|\bar{x}_{i}^{\mathrm{s}}-\bar{x}_{i}^{\mathrm{t}}\right\|_{\infty}$ attained each time instant.

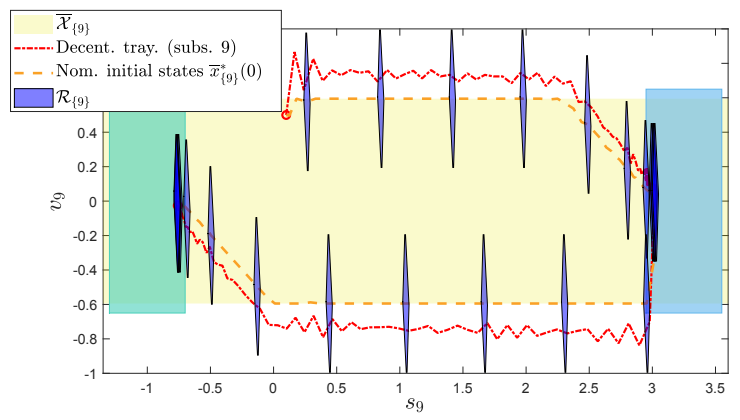

Fig. 7: Invariant tube, nominal initial states, and real evolution of subsystem 9 when using the decentralized topology. For the sake of clarity, the tube is just plotted every eight time steps.

A comparison of the overall performance and communication cumulative costs is provided in Fig. 6. In particular, the cumulative performance costs are measured through the following stage index:

$$
\sum_{i \in \mathcal{N}}\left(\left\|x_{i}-\bar{x}_{i}^{\mathrm{s}}\right\|_{Q_{i}}^{2}+\left\|u_{i}-\bar{u}_{i}^{\mathrm{s}}\right\|_{R_{i}}^{2}+\alpha\left\|\bar{x}_{i}^{\mathrm{s}}-\bar{x}_{i}^{\mathrm{t}}\right\|_{\infty}\right)
$$

while for the communication costs we use $c|\Lambda|$, being $\Lambda$ the active topology each time step. Finally, Fig. 7 shows the invariant tube that characterizes the coupling uncertainty of subsystem 9 when the decentralized topology is imposed.

\section{CONCLUSiOnS}

A robust tube-based MPC strategy for tracking target sets has been introduced in a coalitional MPC framework. In this context, the overall system is controlled through a set of local agents that dynamically merge into clusters to take coordinated decisions. The proposed formulation drives the global system state to the target set in an admissible way, while assuring recursive feasibility and stability regardless the topology switching.

The use of the tracking strategy in conjunction with the coalitional approach allows enlarging the MPC controller domain of attraction without renouncing to the use of decentralized or sparse structures when they suffice for reaching the goals. This fact may be interesting for applications where communications between controllers can fail. In this regard, the dynamic formation of disconnected coalitions limits the information available to the local agents to compute the control 
inputs. To robustify the controller against these uncertainties, the coalitions' constraints set can be restricted. However, it leads to smaller terminal sets and, consequently, reduces the domain of attraction of all noncentralized topologies.

Future research should include the implementation of the proposed approach in more complex multi-agents systems and the use of game-theoretic tools to measure the benefits brought by the agents involved in each coalition.

\section{REFERENCES}

[1] R. Scattolini, "Architectures for distributed and hierarchical model predictive control - A review," Journal of Process Control, vol. 19, no. 5, pp. 723-731, May 2009.

[2] A. Jain, A. Chakrabortty, and E. Biyik, "Distributed wide-area control of power system oscillations under communication and actuation constraints," Control Engineering Practice, vol. 74, pp. 132-143, May 2018.

[3] Y. Wei, S. Li, and Y. Zheng, "Enhanced information reconfiguration for distributed model predictive control for cyber-physical networked systems," International Journal of Robust and Nonlinear Control, vol. 30, no. 1, pp. 198-221, January 2020.

[4] J. Barreiro-Gomez, C. Ocampo-Martinez, and N. Quijano, "Timevarying partitioning for predictive control design: Density-games approach," Journal of Process Control, vol. 75, pp. 1-14, March 2019.

[5] S. Riverso, M. Farina, and G. Ferrari-Trecate, "Plug-and-play model predictive control based on robust control invariant sets," Automatica, vol. 50, no. 8, pp. 2179-2186, August 2014.

[6] S. Riverso, F. Boem, G. Ferrari-Trecate, and T. Parisini, "Plug-and-play fault-detection and control reconfiguration for a class of nonlinear largescale constrained systems," IEEE Transactions on Automatic Control, vol. 61, no. 12, pp. 3963-3978, December 2016.

[7] A. La Bella, P. Klaus, G. Ferrari-Trecate, and R. Scattolini, "Supervised model predictive control of large-scale electricity networks via clustering methods," Optimal Control Applications and Methods, 2021.

[8] J. M. Maestre, D. Muñoz de la Peña, A. Jiménez Losada, E. Algaba, and E. F. Camacho, "A coalitional control scheme with applications to cooperative game theory," Optimal Control Applications and Methods, vol. 35, no. 5, pp. 592-608, September/October 2014.

[9] F. J. Muros, E. Algaba, J. M. Maestre, and E. F. Camacho, "Harsanyi power solutions in coalitional control systems," IEEE Transactions on Automatic Control, vol. 62, no. 7, pp. 3369-3381, July 2017.

[10] A. Nedić and D. Bauso, "Dynamic coalitional TU games: Distributed bargaining among players' neighbors," IEEE Transactions on Automatic Control, vol. 58, no. 6, pp. 1363-1376, June 2013.

[11] F. Fele, J. M. Maestre, and E. F. Camacho, "Coalitional control: Cooperative game theory and control," IEEE Control Systems Magazine, vol. 37, no. 1, pp. 53-69, February 2017.

[12] F. Fele, J. M. Maestre, M. H. Shahdany, D. Muñoz de la Peña, and E. F. Camacho, "Coalitional model predictive control of an irrigation canal," Journal of Process Control, vol. 24, no. 4, pp. 314-325, April 2014.

[13] P. Chanfreut, J. M. Maestre, and E. F. Camacho, "Coalitional model predictive control on freeways traffic networks," IEEE Transactions on Intelligent Transportation Systems, vol. 22, no. 11, pp. 6772-6783, November 2021.

[14] M. Nayeripour, H. Fallahzadeh-Abarghouei, E. Waffenschmidt, and S. Hasanvand, "Coordinated online voltage management of distributed generation using network partitioning," Electric Power Systems Research, vol. 141, pp. 202-209, December 2016.

[15] S. Siniscalchi-Minna, F. D. Bianchi, C. Ocampo-Martinez, J. L. Domínguez-García, and B. De Schutter, "A non-centralized predictive control strategy for wind farm active power control: A wake-based partitioning approach," Renewable Energy, vol. 150, pp. 656-669, 2020.

[16] J. M. Maciejowski, Predictive control: with constraints. Pearson education, 2002

[17] G. Pannocchia and E. Kerrigan, "Offset-free receding horizon control of constrained linear systems," AIChE Journal, vol. 51, no. 12, pp. 3134 3146, December 2005.

[18] D. Mayne, "Model predictive control: Recent developments and future promise," Automatica, vol. 50, no. 12, pp. 2967-2986, December 2014

[19] L. Chisci and G. Zappa, "Dual mode predictive tracking of piecewise constant references for constrained linear systems," International Journal of Control, vol. 76, no. 1, pp. 61-72, 2003.

[20] A. Bemporad, A. Casavola, and E. Mosca, "Nonlinear control of constrained linear systems via predictive reference management," IEEE Trans. on Automatic Control, vol. 42, no. 3, pp. 340-349, March 1997.
[21] E. Gilbert and I. Kolmanovsky, "Nonlinear tracking control in the presence of state and control constraints: a generalized reference governor," Automatica, vol. 38, no. 12, pp. 2063-2073, December 2002.

[22] E. Garone, S. Di Cairano, and I. Kolmanovsky, "Reference and command governors for systems with constraints: A survey on theory and applications," Automatica, vol. 75, pp. 306-328, January 2017.

[23] G. Pannocchia, "Robust model predictive control with guaranteed setpoint tracking," Journal of Process Control, vol. 14, no. 8, pp. 927-937, December 2004.

[24] D. Limon, I. Alvarado, T. Alamo, and E. F. Camacho, "MPC for tracking piecewise constant references for constrained linear systems," Automatica, vol. 44, no. 9, pp. 2382-2387, September 2008.

[25] A. Ferramosca, D. Limon, I. Alvarado, T. Alamo, and E. F. Camacho, "MPC for tracking with optimal closed-loop performance," Automatica, vol. 45, no. 8, pp. 1975-1978, August 2009.

[26] A. Ferramosca, D. Limon, A. H. González, D. Odloak, and E. F Camacho, "MPC for tracking zone regions," Journal of Process Control, vol. 20, no. 4, pp. 506-516, April 2010.

[27] A. Ferramosca, D. Limon, I. Alvarado, and E. F. Camacho, "Cooperative distributed MPC for tracking," Automatica, vol. 49, no. 4, pp. 906-914, April 2013.

[28] M. Razzanelli and G. Pannocchia, "Parsimonious cooperative distributed MPC for tracking piece-wise constant setpoints," in Proc. of the $11^{\text {th }}$ IFAC Symp. on Dynam. and Control of Process Systems, inc. Biosystems (DYCOPS-CAB 2016), Trondheim, Norway, June 2016, pp. 520-525.

[29] D. Limon, I. Alvarado, T. Alamo, and E. F. Camacho, "Robust tubebased MPC for tracking of constrained linear systems with additive disturbances," Journal of Process Control, vol. 20, no. 3, pp. 248-260, March 2010.

[30] W. Langson, I. Chryssochoos, S. Raković, and D. Mayne, "Robust model predictive control using tubes," Automatica, vol. 40, no. 1, pp. 125-133, January 2004.

[31] D. Mayne, M. Seron, and S. Raković, "Robust model predictive control of constrained linear systems with bounded disturbances," Automatica, vol. 41, no. 2, pp. 219-224, February 2005.

[32] P. A. Trodden and P. R. Baldivieso-Monasterios, "Low-complexity robust decentralized MPC: a foundational algorithm for constrained coalitional control," in Proceedings of the $58^{\text {th }}$ Conference on Decision and Control (CDC 2019), Nice, France, December 2019, pp. 1089-1095.

[33] P. R. Baldivieso-Monasterios and P. A. Trodden, "Coalitional predictive control: consensus-based coalition forming with robust regulation," Automatica, vol. 125, p. 109380, March 2021.

[34] K. Wang, A. Michel, and D. Liu, "Necessary and sufficient conditions for the Hurwitz and Schur stability of interval matrices," IEEE Transactions on Automatic Control, vol. 39, no. 6, pp. 1251-1255, June 1994.

[35] D. Mayne and W. Langson, "Robustifying model predictive control of constrained linear systems," Electronic Letters, vol. 37, no. 23, pp. 14221423, November 2001

[36] S. V. Raković, E. C. Kerrigan, K. I. Kouramas, and D. Q. Mayne, "Invariant approximations of the minimal robust positively invariant set," IEEE Trans. on Autom. Control, vol. 50, no. 3, pp. 406-410, Mar. 2005

[37] S. V. Raković and Q. Cheng, "Homothetic tube MPC for constrained linear difference inclusions," in Proc. of the $25^{\text {th }}$ Chinese Control and Decision Conf. (CCDC 2013), Guiyang, China, May 2013, pp. 754-761.

[38] J. Fleming, B. Kouvaritakis, and M. Cannon, "Robust tube MPC for linear systems with multiplicative uncertainty," IEEE Transactions on Automatic Control, vol. 60, no. 4, pp. 1087-1092, April 2015.

[39] M. Bujarbaruah, U. Rosolia, Y. R. Stürz, X. Zhang, and F. Borrelli, "Robust MPC for LTI systems with parametric and additive uncertainty: A novel constraint tightening approach," Submitted to Automatica, 2020.

[40] P. A. Trodden and J. M. Maestre, "Distributed predictive control with minimization of mutual disturbances," Automatica, vol. 77, pp. 31-43, March 2017.

[41] S. Riverso and G. Ferrari-Trecate, "Tube-based distributed control of linear constrained systems," Automatica, vol. 48, no. 11, pp. 2860-2865, November 2012.

[42] P. Gahinet, A. S. Nemirovskii, A. J. Laub, and M. Chilali, LMI Control Toolbox For Use with MATLAB ${ }^{\circledR}$, The MathWorks, Inc., Natick, Massachusetts, USA, 1995.

[43] P. Trodden, "A one-step approach to computing a polytopic robust positively invariant set," IEEE Transactions on Automatic Control, vol. 61, no. 12, pp. 4100-4105, December 2016.

[44] M. Herceg, M. Kvasnica, C. Jones, and M. Morari, "Multi-parametric toolbox 3.0," in Proceedings of the $12^{\text {th }}$ European Control Conference (ECC 2013), Zurich, Switzerland, July 2015, pp. 502-510. 